Bull. Korean Math. Soc. 50 (2013), No. 2, pp. 537-542

http://dx.doi.org/10.4134/BKMS.2013.50.2.537

\title{
ADDENDUM AND ERRATUM TO "ON THE STRUCTURE OF THE FUNDAMENTAL GROUP OF MANIFOLDS WITH POSITIVE SCALAR CURVATURE"
}

\author{
Jin Hong KIm
}

After the publication of the paper [6], it has been noticed that there is an incomplete argument in Lemma 3.2. So we would like to first correct one of the main results of [6] (Theorem 1.2) and then have an opportunity to add some more related results (Theorem 1 below).

As remarked in Remark 2.2 of [6], if the dimension of the manifold $M$ is four, then the scalar curvature of $N_{\alpha}$ can be made positive after a suitable conformal change. Since $N_{\alpha}$ is compact, this implies that the scalar curvature of $N_{\alpha}$ is bounded from below by the constant $k>0$. Thus the scalar curvature of the universal cover $\bar{N}_{\alpha}$ with respect to the pullback metric is again bounded from below by $k$. But then a result of Gromov-Lawson or Schoen-Yau says that the homotopy fill radius of $\bar{N}_{\alpha}$ is bounded from above. So Theorem 1.2 in Section 4 holds to be true in this case without any further condition. On the other hand, if the dimension of $M$ is greater than 4 and less than or equal to 7 , then at the moment we need to add one of the following extra conditions to Theorem 1.2: $\bar{N}_{\alpha}$ has the bounded homotopy fill radius or the self-adjoint elliptic operator $\overline{\mathcal{L}}=-\Delta_{\bar{N}_{\alpha}}+\frac{n-3}{4(n-2)} R_{\bar{N}_{\alpha}}$ is positive-definite on the universal cover $\bar{N}_{\alpha}$ of $N_{\alpha}$. Actually the latter implies the former, as shown in Theorem 3.1 of the paper [6].

Recently we have also obtained an interesting result which is closely related to the main results of the paper [6] (refer to the paper [5] for more detailed accounts). To be precise, our result is stated as follows.

Theorem 1. Let $M$ be a closed oriented Riemannian manifold of dimension 4 with positive isotropic curvature. Then the fundamental group of $M$ does not contain a subgroup isomorphic to the fundamental group of a compact Riemann surface of genus $\geq 2$.

This extends previous results of Fraser [3], Fraser and Wolfson [4], and Brendle-Schoen [1] to the case of a closed oriented Riemannian manifold of

Received November 14, 2011.

2010 Mathematics Subject Classification. 53C20.

Key words and phrases. positive scalar curvature, positive isotropic curvature, fundamental group. 
dimension 4 with positive isotropic curvature and a compact Riemann surface with genus $\geq 2$. The proof of Theorem 1 is achieved by essentially adapting their proofs to the case of a compact Riemann surface with genus $\geq 2$, and we provide it here for the sake of reader's convenience. We also remark that in their paper [2] B.-L. Chen, S.-H. Tang, and X.-P. Zhu announced a complete classification of closed oriented Riemannian manifolds of dimension 4 with positive isotropic curvature which would affirmatively answer the conjecture of Gromov and so Theorem 1 (refer to Conjecture 1.1 of the paper [6]). However, as far as we know, their result has not been published anywhere, yet, and the method of the proof of Theorem 1 is completely different from theirs.

Proof of Theorem 1. For the proof, we suppose that $\pi_{1}(M)$ contains a subgroup $G$ which is isomorphic to $\pi_{1}$ of a compact Riemann surface $\Sigma_{0}$ of genus $g_{0} \geq 2$. Then we will derive a contradiction. The following lemma plays a crucial role.

Lemma 2 (Theorem 1.1 in [4]). Given any $C>0$, there is an integer $k$ and $a$ normal subgroup $N(k, C)$ of $G$ with index $k$ such that

(1) there is a smooth map $h_{k, C}: \Sigma \rightarrow M$ of a compact Riemann surface $\Sigma$ into $M$ satisfying the property that

$$
\left(h_{k, C}\right)_{*}: \pi_{1}(\Sigma) \rightarrow \pi_{1}(M)
$$

is injective onto $N(k, C)$,

(2) for any such $h_{k, C}$, every closed non-trivial geodesic $\gamma$ on $\Sigma$ has length $>C$ with respect to the induced metric by $h_{k, C}$.

If we carefully look at the proof of Lemma 2 (or Theorem 1.1 in [4]), then one can easily see that as $C$ goes to infinity, so does $k$. For the proof of Theorem 1 , we will also need the following lemma.

Lemma 3 (Theorem 1.2 in [4]). If every closed non-trivial geodesic $\gamma$ on a compact Riemann surface $\Sigma$ has length $>C$, then there is a Lipschitz distance decreasing degree-one map $f: \Sigma \rightarrow S^{2}$ such that $C|d f| \leq D$, where $D$ is some constant independent of $C$.

Now, we may assume that $M$ has positive isotropic curvature $\geq \kappa>0$, since $M$ is compact. Then fix a positive constant $C>0$ which will be chosen explicitly later. Let $h_{0}: \Sigma_{0} \rightarrow M$ be a smooth map such that $\left(h_{0}\right)_{*}: \pi_{1}\left(\Sigma_{0}\right) \rightarrow$ $\pi_{1}(M)$ is an isomorphism onto $G$. Then it follows from Lemma 2 that there are a compact Riemann surface $\Sigma$ of genus $g$, a regular $k$-covering $p: \Sigma \rightarrow \Sigma_{0}$, and a smooth map $h_{k, C}: \Sigma \rightarrow M$ given by $h_{k, C}=h_{0} \circ p$ such that $\left(h_{k, C}\right)_{*}: \pi_{1}(\Sigma) \rightarrow$ $\pi_{1}(M)$ is injective with $\left(h_{k, C}\right)_{*}\left(\pi_{1}(M)\right)=: N(k, C)$ a normal subgroup of $G$ of index $k$. For simplicity, let $h=h_{k, C}$. Note that the Euler characteristic $\chi(\Sigma)$ is equal to $k$ times of the Euler characteristic $\chi\left(\Sigma_{0}\right)$ of $\Sigma_{0}$, so we obtain $2-2 g=k\left(2-2 g_{0}\right)$ and thus $g=k\left(g_{0}-1\right)+1 \geq 1$. Note also from the proof of Lemma 2 that for every map $\tilde{h}: \Sigma \rightarrow M$ whose induced map $\tilde{h}_{*}$ on $\pi_{1}(\Sigma)$ equals $h_{*}$, every closed non-trivial geodesic $\gamma$ has length $>C$ with 
respect to the induced metric by $\tilde{h}$. Since $h$ is incompressible in the sense that $h_{*}: \pi_{1}(\Sigma) \rightarrow \pi_{1}(M)$ is injective, it follows from a theorem of Schoen and Yau that there is a stable conformal branched minimal immersion $u: \Sigma \rightarrow M$ such that $u_{*}=h_{*}$ on $\pi_{1}(\Sigma)$.

Let $F$ denote the pull-back of the normal bundle $\mathcal{N}$ of the minimal surface $u(\Sigma)$ equipped with the pull-back metric and normal connection $\nabla^{\perp}$. Then it is known that $F$ is a smooth vector bundle of real rank 2 over $\Sigma$, even across the branch points (see p. 8 of [1]). Let $E$ be the complexification of $F$, so $E=F \otimes \mathbf{C}$. Since $E$ is a complexification of a real bundle $F$ which is isomorphic to its dual bundle $F, E$ is isomorphic to its dual bundle $E^{*}$ so that we have $c_{1}(E)=0$. The metric on $F$ extends as a complex bilinear form $($,$) on E$ or as a Hermitian metric $\langle$,$\rangle on E$. So the connection $\nabla^{\perp}$ and the curvature form extend complex linearly to sections $s$ of $E$. Then there is a unique holomorphic structure on $E$ such that the $\bar{\partial}$ operator is given by

$$
\bar{\partial} s=\left(\nabla_{\frac{\partial}{\partial \bar{z}}}^{\perp} s\right) d \bar{z}
$$

where $x$ and $y$ are local coordinates on $\Sigma$ and $\frac{\partial}{\partial \bar{z}}=\frac{1}{2}\left(\frac{\partial}{\partial x}+i \frac{\partial}{\partial y}\right)$. Moreover, $E$ splits as a direct sum of a holomorphic line bundle $E^{(1,0)}$ and an antiholomorphic line bundle $E^{(0,1)}$. Hence we have

$$
0=c_{1}(E)=c_{1}\left(E^{(1,0)}\right)+c_{1}\left(E^{(0,1)}\right) .
$$

This implies that we may assume without loss of generality that $c_{1}\left(E^{(1,0)}\right) \geq 0$.

Recall that, by using the complexified formula for the second variation of area, the stability condition can be stated as

$$
\int_{\Sigma}\left(\left|\nabla_{\frac{\partial}{\partial z}}^{\perp} s\right|^{2}-\left|\nabla_{\frac{\partial}{\partial z}}^{T} s\right|^{2}\right) d x d y \geq \int_{\Sigma}\left\langle R\left(s, \frac{\partial u}{\partial z}\right) \frac{\partial u}{\partial \bar{z}}, s\right\rangle d x d y
$$

for all $s$ in the space of sections $\Gamma(E)$ (see [3] and [4]). Notice that every section $s \in \Gamma\left(E^{(1,0)}\right)$ is isotropic in the sense that $(s, s)=0$. Since $M$ has positive isotropic curvature, it follows from (0.1) that we obtain

$$
\int_{\Sigma}\left|\nabla_{\frac{\partial}{\partial z}}^{\perp} s\right|^{2} d x d y \geq \int_{\Sigma} \kappa\left|\frac{\partial u}{\partial z}\right|^{2}|s|^{2} d x d y
$$

for all $s \in \Gamma\left(E^{(1,0)}\right)$. By using the inequality $(0.2)$ and the fact that $u$ is nonconstant, one can also show that there is a positive constant $\varepsilon=\varepsilon(C)$ such that

$$
\int_{\Sigma}\left|\nabla_{\frac{\partial}{\partial z}}^{\perp} s\right|^{2} d x d y+\frac{1}{2} \int_{\Sigma} \kappa\left|\frac{\partial u}{\partial z}\right|^{2}|s|^{2} d x d y \geq \frac{\kappa \varepsilon}{2} \int_{\Sigma}|s|^{2} d x d y
$$

for all $s \in \Gamma\left(E^{(1,0)}\right)$. Indeed, suppose that for every $\varepsilon>0$ we have

$$
\int_{\Sigma}\left|\nabla \frac{\partial}{\partial z} s\right|^{2} d x d y+\frac{1}{2} \int_{\Sigma} \kappa\left|\frac{\partial u}{\partial z}\right|^{2}|s|^{2} d x d y<\frac{\kappa \varepsilon}{2} \int_{\Sigma}|s|^{2} d x d y .
$$


Then it follows from (0.2) and (0.4) that we have

$$
\begin{aligned}
\frac{3}{2} \kappa \int_{\Sigma}\left|\frac{\partial u}{\partial z}\right|^{2}|s|^{2} d x d y & \leq \int_{\Sigma}\left|\nabla_{\frac{\partial}{\partial z}}^{\perp} s\right|^{2} d x d y+\frac{1}{2} \kappa \int_{\Sigma}\left|\frac{\partial u}{\partial z}\right|^{2}|s|^{2} d x d y \\
& <\frac{\kappa \varepsilon}{2} \int_{\Sigma}|s|^{2} d x d y
\end{aligned}
$$

Let $t=\frac{s}{\left(\int_{\Sigma}|s|^{2} d x d y\right)^{1 / 2}}$. Thus we obtain

$$
\int_{\Sigma}\left|\frac{\partial u}{\partial z}\right|^{2}|t|^{2} d x d y<\frac{\varepsilon}{3}
$$

for any $\varepsilon>0$. Since $|t|^{2}$ is not zero everywhere and $\left|\frac{\partial u}{\partial z}\right|=\left|\frac{\partial u}{\partial \bar{z}}\right|$, we have $\left|\frac{\partial u}{\partial z}\right|^{2}=0$ and so $\frac{\partial u}{\partial z}=0$ and $\frac{\partial u}{\partial \bar{z}}=0$. This implies that $u$ is constant, which is clearly a contradiction.

Now, taking the arithmetic mean of $(0.2)$ and $(0.3)$, we easily obtain

$$
\int_{\Sigma}\left|\nabla_{\frac{\partial}{\partial z}}^{\perp} s\right|^{2} d x d y \geq \frac{\kappa}{4} \int_{\Sigma}\left(\left|\frac{\partial u}{\partial z}\right|^{2}+\varepsilon\right)|s|^{2} d x d y
$$

for all $s \in \Gamma\left(E^{(1,0)}\right)$.

Next, we define a new Riemannian metric $\tilde{g}$ on $\Sigma$ by

$$
\tilde{g}=u^{*} g+2 \varepsilon(d x \otimes d x+d y \otimes d y)=u^{*} g+\varepsilon(d z \otimes d \bar{z}+d \bar{z} \otimes d z) .
$$

Then every closed non-trivial geodesic on $\Sigma$ has length $>C$ with respect to $\tilde{g}$. So it follows from Lemma 3 that there is a Lipschitz distance decreasing map $f: \Sigma \rightarrow S^{2}$ of degree one with

$$
C|d f| \leq D,
$$

where $D$ is a constant independent of $f$. Hence we have

$$
C^{2}\left|\frac{\partial f}{\partial z}\right|^{2} \leq D\left|\frac{\partial}{\partial z}\right|_{\tilde{g}}^{2}=D\left(\left|\frac{\partial u}{\partial z}\right|^{2}+\varepsilon\right) .
$$

Let $\xi$ be a holomorphic line bundle over $S^{2}$ with $c_{1}(\xi)>g-1$, where $g$ is the genus of $\Sigma$. Fix a metric and a connection on $\xi$, and choose sections $\alpha_{1}$ and $\alpha_{2}$ in $\Gamma\left(\xi^{*}\right)$ such that $\left|\alpha_{1}\right|+\left|\alpha_{2}\right| \geq 1$ on $S^{2}$. Let $\tilde{\xi}=f^{*} \xi$. Then we have $c_{1}(\tilde{\xi})>g-1$. Since $c_{1}\left(E^{(1,0)}\right) \geq 0$, we also have $c_{1}\left(E^{(1,0)} \otimes \tilde{\xi}\right) \geq c_{1}(\tilde{\xi})>g-1$. By the Riemann-Roch theorem, we have

$$
\begin{aligned}
h^{0}\left(\Sigma, E^{(1,0)} \otimes \tilde{\xi}\right) & =h^{1}\left(\Sigma, E^{(1,0)} \otimes \tilde{\xi}\right)+c_{1}\left(E^{(1,0)} \otimes \tilde{\xi}\right)-g+1 \\
& \geq c_{1}(\tilde{\xi})-g+1>0,
\end{aligned}
$$

where $h^{j}\left(\Sigma, E^{(1,0)} \otimes \tilde{\xi}\right)(j=0,1)$ denotes the complex dimension of the Dolbeaut cohomology $H^{j}\left(\Sigma, E^{(1,0)} \otimes \tilde{\xi}\right)$. This implies that there is a non-vanishing holomorphic section $\sigma$ on $E^{(1,0)} \otimes \tilde{\xi}$.

For each $j=1,2$, set $\tau_{j}=f^{*} \alpha_{j} \in \Gamma\left(\tilde{\xi}^{*}\right)$ and $s_{j}=\sigma \otimes \tau_{j} \in \Gamma\left(E^{(1,0)}\right)$. Since $\sigma$ is holomorphic, we obtain $\nabla_{\frac{\partial}{\partial \bar{z}}}^{\perp} s_{j}=\sigma \otimes \nabla_{\frac{\partial}{\partial \bar{z}}} \tau_{j}$ and $\left|\nabla_{\frac{\partial}{\partial \bar{z}}} \tau_{j}\right|^{2}=\left|\nabla_{\frac{\partial f}{\partial z}} \alpha_{j}\right|^{2} \leq$ 
$C^{\prime}\left|\frac{\partial f}{\partial z}\right|^{2}$, where $C^{\prime}$ is a positive constant independent of $k$ and $C$. Hence we have

$$
\left|\nabla_{\frac{\partial}{\partial z}}^{\perp} s_{j}\right|^{2}=|\sigma|^{2}\left|\nabla_{\frac{\partial}{\partial z}} \tau_{j}\right|^{2} \leq C^{\prime}|\sigma|^{2}\left|\frac{\partial f}{\partial z}\right|^{2}
$$

for $j=1,2$. If we combine the inequality (0.6) with (0.7), we obtain

$$
C^{2}\left|\nabla_{\frac{\partial}{\partial \bar{z}}}^{\perp} s_{j}\right|^{2} \leq C^{2} C^{\prime}|\sigma|^{2}\left|\frac{\partial f}{\partial z}\right|^{2} \leq C^{\prime} D|\sigma|^{2}\left(\left|\frac{\partial u}{\partial z}\right|^{2}+\varepsilon\right) .
$$

On the other hand, it is easy to see

$$
C^{2} \int_{\Sigma}\left(\left|\nabla_{\frac{\partial}{\partial \bar{z}}}^{\perp} s_{1}\right|^{2}+\left|\nabla_{\frac{\partial}{\partial \bar{z}}}^{\perp} s_{2}\right|^{2}\right) d x d y \leq 2 C^{\prime} D \int_{\Sigma}|\sigma|^{2}\left(\left|\frac{\partial u}{\partial z}\right|^{2}+\varepsilon\right) d x d y .
$$

Since $\left|s_{1}\right|+\left|s_{2}\right|=|\sigma|\left(\left|\tau_{1}\right|+\left|\tau_{2}\right|\right) \geq|\sigma|$ on $\Sigma$, by (0.5) we also have $(0.9)$

$$
\begin{aligned}
\int_{\Sigma}\left(\left|\nabla_{\frac{\partial}{\partial z}}^{\perp} s_{1}\right|^{2}+\left|\nabla_{\frac{\partial}{\partial z}}^{\perp} s_{2}\right|^{2}\right) d x d y & \geq \frac{\kappa}{4} \int_{\Sigma}\left(\left|s_{1}\right|^{2}+\left|s_{2}\right|^{2}\right)\left(\left|\frac{\partial u}{\partial z}\right|^{2}+\varepsilon\right) d x d y \\
& \geq \frac{\kappa}{8} \int_{\Sigma}|\sigma|^{2}\left(\left|\frac{\partial u}{\partial z}\right|^{2}+\varepsilon\right) d x d y
\end{aligned}
$$

By comparing two inequalities (0.8) and (0.9), we have

$$
\begin{aligned}
2 C^{\prime} D \int_{\Sigma}|\sigma|^{2}\left(\left|\frac{\partial u}{\partial z}\right|^{2}+\varepsilon\right) d x d y & \geq C^{2} \int_{\Sigma}\left(\left|\nabla_{\frac{\partial}{\partial \bar{z}}}^{\perp} s_{1}\right|^{2}+\left|\nabla_{\frac{\partial}{\partial z}}^{\perp} s_{2}\right|^{2}\right) d x d y \\
& \geq \frac{\kappa}{8} C^{2} \int_{\Sigma}|\sigma|^{2}\left(\left|\frac{\partial u}{\partial z}\right|^{2}+\varepsilon\right) d x d y
\end{aligned}
$$

Thus it is easy to obtain $2 C^{\prime} D \geq \frac{\kappa}{8} C^{2}$, and so $C$ should be less than or equal to $\left(\frac{16 C^{\prime} D}{\kappa}\right)^{1 / 2}$. Finally, if we take $C>\left(\frac{16 C^{\prime} D}{\kappa}\right)^{1 / 2}$, then we would have a contradiction. This completes the proof of Theorem 1.

Note added in proof: The paper [2] is now published in Journal of Differential Geometry 91 (2012), no. 1, 41-80.

Acknowledgements. This research was supported by Basic Science Research Program through the National Research Foundation of Korea (NRF) funded by the Ministry of Education, Science and Technology (2012-0007404).

\section{References}

[1] S. Brendle and R. Schoen, Sphere theorems in geometry, Surveys in differential geometry. Vol. XIII. Geometry, analysis, and algebraic geometry: forty years of the Journal of Differential Geometry, 49-84, Surv. Differ. Geom., 13, Int. Press, Somerville, MA, 2009.

[2] B.-L Chen, S.-H. Tang, and X.-P. Zhu, Complete classification of compact four-manifolds with positive isotropic curvature, preprint, 2008.; arXiv:0810.1999v1.

[3] A. Fraser, Fundamental groups of manifolds with positive isotropic curvature, Ann. Math. 158 (2003), no. 1, 345-354.

[4] A. Fraser and J. Wolfson, The fundamental group of manifolds of positive isotropic curvature and surface groups, Duke Math. J. 133 (2006), no. 2, 325-334. 
[5] J. H. Kim, Surface groups and four-manifolds with positive isotropic curvature, preprint, 2011.

[6] J. H. Kim and H. C. Park, On the structure of the fundamental group of manifolds with positive scalar curvature, Bull. Korean Math. Soc. 48 (2011), no. 1, 129-140.

Department of Mathematical Sciences

KAIST

DAEJEON 305-701, KOREA

E-mail address: jinhkim11@gmail.com 